\title{
Sleep, Sleep Self-Efficacy Perceptions and Related Correlates in Women with Knee Osteoarthritis-An Exploratory Secondary Analysis
}

\author{
Dr. Ray Marks
}

School of Health Sciences and Professional Studies, City University of New York, York College, and Department of Health \& Behavior Studies, Columbia University, Teachers College, New York, USA.

rm226@columbia.edu

*Corresponding Author: Dr. Ray Marks, Department of Health and Behavior Studies, Columbia University, Teachers College, Box 114, 525W 120th Street, New York, USA.

\begin{abstract}
Older women with knee osteoarthritis, a highly prevalent and debilitating joint disease, often experience greater physical disability, cognitive distress, and excess pain, when compared to men with the same disease. To better understand the factors potentially contributing to this discrepancy, this present retrospective analysis of 18 women with a mean age of 71 years and mild to moderate knee osteoarthritis specifically strove to detail the presence of any consistent sleep disturbance, along with possible clinical correlates, including pain self-efficacy and others on a single test occasion in the absence of intervention. After a series of salient validated survey questionnaires and demographic data forms were completed, they were reduced and subjected to descriptive and correlational analyses. Results showed: 1) Sleep disturbances during the past week occurred in more than $50 \%$ cases to various degrees; 2) Those with lower levels of perceived pain self-efficacy for managing night pain had more frequent episodes of sleep 'restlessness' in the past week ( $r=-.63 ; p=.01) ; 3)$ Pain self-efficacy item scores for sleep were significantly correlated with the individual's overall confidence in their ability to reduce overall pain ( $r=.65 ; p=.01)$, their depressive symptom scores ( $p=.01)$, feeling sad ( $p=.02)$, their visual analogue pain intensity scores ( $p=.01$ ), Arthritis Impact Measurement scores ( $r=.05)$ and feeling depressed ( $p=.03) .4)$ The sleep variable was unaffected by age ( $p=.21)$, or body mass ( $p=.90)$, and had no bearing on ambulatory capacity ( $p=.34$ ) or directly on pain ( $p=.05)$, or the composite pain self-efficacy score ( $p=.05)$. It is concluded a sizeable number of older women with knee osteoarthritis may experience sleep disturbances, and feel less than efficacious in managing night pain, and may hence be at risk for excess pain and depression if intervention is not forthcoming.
\end{abstract}

Keywords: Depression, Disability, Knee Joint, Osteoarthritis, Pain, Self-efficacy, Sleep, Women

\section{INTRODUCTION}

Osteoarthritis, a prevalent disabling joint disease affecting many older adults [1] frequently affects one or more compartments of one or both knee joints [2]. Associated with significant functional disability, joint stiffness and pain [3], among other key physiological impairments [4], the disease is seldom responsive to current non-pharmacologic or oftentimes surgical approaches. A disease with no known cause, nor cure, and one where women are often affected more frequently than men [5-8], as well as more intensely than men [9], research has pointed to the fact that the disease, is not only a physically challenging one, but one commonly compounded by varying degrees of emotional distress, including depression and/or impairments of general health, vitality, and reduced life quality $[5,10,11]$. More recently, the role of negative personal beliefs, as well as sleep disturbances [12] has also been discussed in efforts to target possible osteoarthritis correlates that are amenable to modification.

However, even though numerous studies conducted in the last 30 years have examined some of the aforementioned factors in isolation, very few have 
Sleep, Sleep Self-Efficacy Perceptions and Related Correlates in Women with Knee Osteoarthritis-An Exploratory Secondary Analysis

focused on examining aspects of the disease such as sleep, and none directly assessed if self-efficacy for sleep related management purposes, a dimension of the construct of pain self-efficacy (an individual belief denoting the extent of one's confidence to control various aspects of pain through behavioral and other strategies) may be an important correlate of the disease accounting for the greater disease burden experienced by women with knee osteoarthritis than men and its disabling features in its own right. Since sleep attributes, as well as pain self-efficacy attributes appear to influence osteoarthritis and its symptoms independently, it seemed reasonable to examine if the extent to which patients' perceptions about their ability to cope with their possible sleepassociated limitations would be related to the overall extent of any prevailing sleep disturbances. Since knee osteoarthritis disability is common among community dwelling middle aged and older women, but who may suffer more than men with the same disease presentation [5] this exploratory study chose to focus on establishing if there is any link between the pain experienced by this group, their ability to sleep through the night, and their perceived confidence they could control any emergent night pain. This does not exclude the possibility that these variables are salient among men with knee osteoarthritis, but the target in this study was delimited to women.

To this end, this report describes selected observations compiled from reports of 18 women 59 years of age or older with radiological and clinically verified unilateral or bilateral knee joint osteoarthritis living in the community drawn randomly from a larger sample of similar women. The women were generally considered healthy, none had had surgery, and none were scheduled for surgery. All were deemed eligible to enter a community based exercise associated study.

Among the specific variables of interest reported here are those assessed at baseline, and that included the extent to which these subjects reported having restless sleep issues as described on a 1-4 point Likert scale of increasing duration in the past week (this scale ranges from rarely or none of the time or less than 1 day/week, to most or all of the time or 5-7 days per week. In addition to basic demographic and disease associated features, a validated measure of overall pain self-efficacy expressed as a summative score of 5-item scores ranging from 10-100 (low-high confidence), as well as the item scores were specifically sought

as a previous larger study showed the item related to the subject's perceived self-efficacy for 'overcoming sleep challenges due to pain' to be the most salient in the Arthritis Self Efficacy Pain scale relative to pain. Secondarily, it was of interest to assess if the pain selfefficacy item score mentioned here would have any correspondence to overall pain, depression, pain selfefficacy cumulative scores, function, age, and body mass characteristics, as well as if the degree to which sleep might be disturbed and its association with age, disease status, body mass, and pain.

This particular focus was taken in light of emerging data concerning sleep issues in the context of pain, suffering, as well as the degree to which self-efficacy attributes are related to pain. Women were studied independently because they suffer more than men with this condition and may suggest they have some unique characteristics that are overlooked in more inclusive studies. Since deficits in self-efficacy attributes are generally amenable to behavioral specific-intervention, it was decided to explore item as well as aggregate pain self-efficacy scores, because the item scores may clearly contain unique information.

It was hypothesized that:

a. A sizeable number of the presently observed cohort would report encountering some degree of restless sleep over the course of a regular week and this would range from modest to impactful in severity.

b. Based on prior work, it was anticipated that the extent of the subject's confidence to overcome sleep issues due to pain would be an important pain self-efficacy attribute that would vary inversely with sleep quality perceptions.

c. Those with higher self-efficacy scores for overcoming sleep challenges would have less pain, depression, and more overall confidence to manage their pain.

Since innumerable studies have failed to account for the persistent pain women with knee osteoarthritis have reported experiencing, it was felt results of this exploratory study could help to drive future hypotheses and research. Moreover, the results were expected to inform clinicians about what might prove especially valuable for enhancing functional independence and health related quality of life in women with similar personal characteristics. 
Sleep, Sleep Self-Efficacy Perceptions and Related Correlates in Women with Knee Osteoarthritis-An Exploratory Secondary Analysis

The specific questions of interest were:

1. Do a sizeable number of women with knee osteoarthritis experience one or more sleep disturbances during a regular week?

2. What are possible key sleep correlates among a sample of typical community dwelling women with established radiographic and clinical evidence of knee osteoarthritis?

3. What can the data reveal that will help advance recommendations for reducing the risk of excessive disability among a similar group of women?

\section{Materials AND Methods}

Records of an available sub-sample of 18 communitydwelling women drawn from a larger sample of 47 women with radiological confirmed kneeosteoarthritis of one or both knees were examined. All had either the medial or lateral compartment osteoarthritis of grade II severity, and could walk independently with or without assistive devices.

Excluded were men, women with rheumatoid arthritis, or definitive arthritis of other joints, as were those with any neurological impairment, an uncontrollable or unstable medical condition, or those unable to follow instructions or basic English. All subjects had consented to being assessed for eligibility in an exercise study.

Among the self-reports completed by eligible subjects were items on: The Arthritis Impact Measurement Scale (AIMS)(Physical, Health, Pain and Impact Subscales) [13], The Center for Epidemiological Studies Depression Scale (CES-D)[14], a $10 \mathrm{~cm}$ long Visual Analogue Pain Scale where 0 was no pain [15] and the Arthritis Self-Efficacy for Pain and Other Symptoms Scales [16]. Demographic variables included age, numbers of affected joints, disease duration, comorbid health status, height, and weight. Self-reported sleep disturbances over the last week were reported on a 1-4 point scale where $1=$ rarely or none of the time; $2=1-2$ days; $3=$ occasionally or $3-4$ days; and $4=$ most days or every day, and scored by collapsing the 4 scores according to standard procedures. Standard body weight $(\mathrm{kg})$ and height $(\mathrm{m})$ measures were used to derive a body mass index (w.h $\mathrm{h}_{-2}$ ) for each individual. Depression using the CES-D was estimated by summing the 20-item scores, which could range from
$0-60$, with a cut-off point for depression of 16 . Total distance walked in 6 minutes at self-paced speed on a standardized level indoor walkway, level of exertion following this using Borg Perceived Exertion Scale (0-15), levels of sadness (1-4), and other emotional factors were itemized from the CESD.

The data were entered systematically into an Excel file and then selected descriptive statistics, and inferential tests including correlations that could potentially address the study questions were calculated using SPSS version 23.00. Here, the prevalence of sleep issues, plus demographic and disease correlates, especially cognitive status and perceptions concerning personal efficacy for managing sleep were of key import. The significance level was set at $\mathrm{p}<0.05$, and although the sample was small, a power analysis showed 18 subjects would be sufficient to demonstrate significant interrelationships between the selected demographic, pain and sleep efficacy variables.

\section{RESULTS}

As outlined in Table 1, which portrays the mean and range of scores recorded for the present group as regards the key variables of present interest, this cohort was somewhat typical of functional grade 1 or 2 osteoarthritis disability levels and a group not generally in severe pain, nor suffering to a great degree from depression. However, more than 50\% described sleep challenges in some form that ranged from no disturbances to disturbances 4-7 nights per week, regardless of whether they suffered from unilateral knee joint osteoarthritis, or bilateral knee joint osteoarthritis, with slightly more challenges for those with bilateral joint disease (see Figure 1). Those who reported one or more weekly disturbances of sleep, did not appear to have distinctive age, or disease duration profiles, but tended to have less confidence to secure a good nights' sleep without pain, as well as in their ability to reduce pain specifically. Sleep was slightly more affected if subjects were categorized as having a cardiovascular health condition (mean: 1.67, CI .40-2.9' verses 2.00, CI 1.17-2.82, or one or more comorbid health conditions, or higher pain levels. Pain self-efficacy scores as regards sleeping with pain, which appeared to be the strongest correlate of the sleep data, ranged from 10-100 with a mean of $72.35 \pm 26.35$ implying a general lack of optimal confidence in this respect. That is, subjects who were 
Sleep, Sleep Self-Efficacy Perceptions and Related Correlates in Women with Knee Osteoarthritis-An Exploratory Secondary Analysis

generally less than confident about their ability to optimally limit or reduce their pain experience during sleep periods reported slightly more weekly sleep challenges than those classified as having high self efficacy for reducing night pain (1.78 vs 2.00 mean scores). The former also tended to have more pain, higher depression scores, and lower overall pain self efficacy scores, and less confidence to deal with fatigue, regardless of age. At the same time, those categorized as having depression had a much increased chance of reporting sleep challenges, as did those with modest pain self-efficacy levels, those with 'good' pain selfefficacy rating scores reported fewer sleep incidents than those with limited pain self-efficacy, along with those who had pain in two or more joints, or were in fair versus 'excellent' health [see Tables 2 and 3].

Table 1. Basic Descriptive Characteristics of Knee Osteoarthritis Cohort ( $N=18)$.

\begin{tabular}{|c|c|}
\hline Variable & Statistics \\
\hline \multicolumn{2}{|l|}{ Age (Years) } \\
\hline Range & $59-84$ \\
\hline $\mathrm{Mean} \pm \mathrm{SD}$ & $70.11 \pm 7.5$ \\
\hline \multicolumn{2}{|l|}{ Disease duration (Years) } \\
\hline Range & $4-50$ \\
\hline Mean+SD & $12.83 \pm 11.3$ \\
\hline \multicolumn{2}{|l|}{ Morbidity count $\{\mathrm{N}\}$} \\
\hline Range & $0-4$ \\
\hline $\mathrm{Mean} \pm \mathrm{SD}$ & $1 \pm 1.1$ \\
\hline \multicolumn{2}{|l|}{ Body mass index (h.w-2)(BMI) } \\
\hline Range & $23.5-33.2$ \\
\hline $\mathrm{Mean} \pm \mathrm{SD}$ & $27.9 \pm 2.9$ \\
\hline \multicolumn{2}{|l|}{ Depressive Symptoms (CESD) } \\
\hline Range & $0-25$ \\
\hline Mean+SD & $9.39 \pm 6.9$ \\
\hline \multicolumn{2}{|c|}{ AIMS Impact Score 0-10 Rating Scale } \\
\hline Range & $0-7.5$ \\
\hline $\mathrm{Mean} \pm \mathrm{SD}$ & $3.61 \pm 2.6$ \\
\hline \multicolumn{2}{|c|}{ VAS Pain Score 0-10 Rating Scale } \\
\hline Range & $1.0-10.0$ \\
\hline $\mathrm{Mean} \pm \mathrm{SD}$ & $4.6 \pm 3.0$ \\
\hline \multicolumn{2}{|c|}{ Perceived Exertion 0-15 Borg Rating } \\
\hline Range & $9-14$ \\
\hline Mean \pm SD & $11.3 \pm 1.7$ \\
\hline \multicolumn{2}{|c|}{ *Pain Self Efficacy 5 Point 10-100 Scale } \\
\hline Range & $34-100$ \\
\hline Mean \pm SD & $73.9 \pm 19.03$ \\
\hline \multirow[t]{2}{*}{ *Item 3Pain Self Efficacy-Sleep } & $10-100$ \\
\hline & $73.9 \pm 26.4$ \\
\hline \multicolumn{2}{|c|}{ Sleep score 1-4 Point Numeric Scale } \\
\hline Range & $1.0-4.0$ \\
\hline Mean+SD & $1.94 \pm 1.06$ \\
\hline
\end{tabular}


Sleep, Sleep Self-Efficacy Perceptions and Related Correlates in Women with Knee Osteoarthritis-An Exploratory Secondary Analysis

Table 2. Significant Sleep Related Pearson Product Moment Correlates Among Cohort $(N=16)(p<0.05)$.

\begin{tabular}{lllll}
\hline Restless Sleep & Pain & Pain SE & PSE1 & PSE3 \\
$\mathbf{( 1 - 4 )}$ & VAS (1-10) & ASES (10-100) & & \\
\hline $\mathrm{r}$ & .502 & -.478 & -.503 & .778 \\
$\mathrm{p}$ & .040 & .052 & .040 & .001 \\
\hline
\end{tabular}

Abbreviations: ASES=arthritis self-efficacy scale; PSE1; Pain Self efficacy Item 1; PES2; Pain Self-efficacy Item 3; VAS visual analogue pain scale

Table 3. Selected Zero Order Correlates between Pain Self Efficacy for Sleep Scores and Key Functional and Cognitive Measures ( $N=18)$.

\begin{tabular}{llllll}
\hline Night Pain SE Score & Disease Impact & Pain & Depression & Pain SE & Borg Exertion Score \\
ASES Pain Item 3 & AIMS & VAS & CESD & ASES Pain & $\mathbf{( 1 - 1 5 )}$ \\
$\mathbf{( 1 0 - 1 0 0 )}$ & $\mathbf{( 1 - 1 0 )}$ & $\mathbf{( 1 - 1 0 )}$ & $\mathbf{( 0 - 6 0 )}$ & $\mathbf{( 1 0 - 1 0 0 )}$ & \\
\hline $\mathrm{r}$ &.- .473 & -.540 & -.631 & .706 & -.398 \\
$\mathrm{p}$ & .047 & .021 & .005 & .001 & .102 \\
\hline
\end{tabular}

Abbreviations: $\mathrm{AI}<\mathrm{S}=$ arthritis impact; $\mathrm{ASES}=$ arthritis self-efficacy pain score; $\mathrm{CESD}=$ depression scale score.

Table 4. Sample of Data from a Subject 72 Years- with Left sided knee OA-Tracked over One Year

\begin{tabular}{lccccc}
\hline Time & $\begin{array}{l}\text { Sleep Rating } \\
(\mathbf{1 - 4 )}\end{array}$ & $\begin{array}{l}\text { PSE3 } \\
\mathbf{( 1 0 - 1 0 0 )}\end{array}$ & $\begin{array}{l}\text { Borg Score } \\
\mathbf{( 1 - 1 5 )}\end{array}$ & $\begin{array}{l}\mathbf{6} \text { min walk } \\
\mathbf{f t} .\end{array}$ & $\begin{array}{l}\text { Weight } \\
\text { lbs }\end{array}$ \\
\hline 1 & 1 & 80 & 12 & 1194 & $127 \mathrm{lb}$ \\
2 & 2 & 70 & 13 & 1168 & $130 \mathrm{lb}$ \\
\hline
\end{tabular}

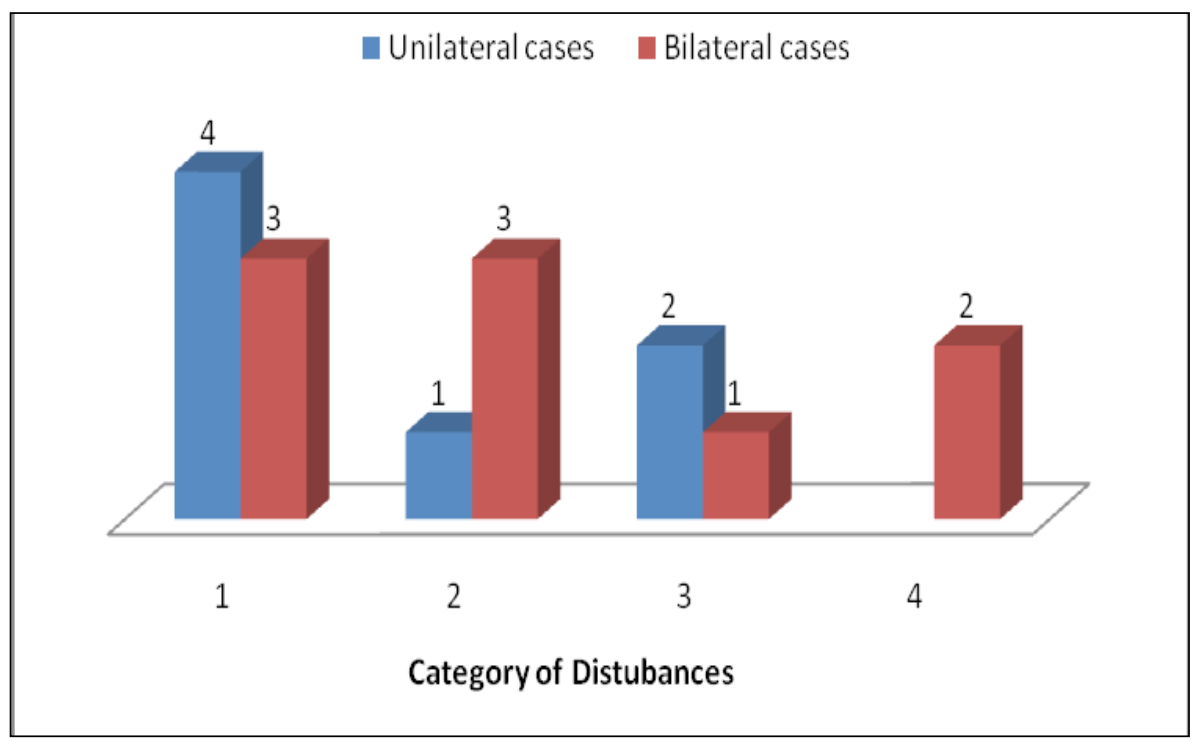

Figure 1. Numbers Reporting Restiess Nights/Past Week 
Sleep, Sleep Self-Efficacy Perceptions and Related Correlates in Women with Knee Osteoarthritis-An Exploratory Secondary Analysis

\section{DISCUSSION}

Osteoarthritis of the knee, a progressive joint disease commonly produces a multi-dimensional clinical syndrome, which often includes intractable symptoms of pain, fatigue, functional, occupational and social limitations, especially in women. Associated with a variety of pathological changes such as joint instability and malalignment, as well as psychological symptoms such as anxiety and depression, the disease can progressively impair the musculoskeletal functioning and life quality of the sufferer quite significantly.

However, despite years of research very little progress has been made to understand the interaction of underlying disease symptoms as this relates to excess disability prevention as well as treatment of this condition. While the role of obesity and aging has been studied to some degree, cognitions and sleep related problems that may contribute to obesity as well as the ambulatory limitations among this patient group, along with self-efficacy attributes that may influence osteoarthritis disability and depression among other factors are relatively unexplored among samples of women who are generally found to suffer more from this disease than men and have differing self-efficacy profiles $[18,19]$.

In the present exploratory case analysis, which was restricted to active community dwelling older women with verifiable knee osteoarthritis of one or both joints, the focus was placed on the association between affective subjective responses of pain, depression, pain related sleep efficacy and sleep disturbances-a possible correlate of fatigue, overweight, activity limitations. The operational definition used to include or exclude potential study candidates required all to have radiographic and clinically verifiable disease as identified by a physician of one or both knees.

To support the view that age is only one variable that may influence the natural history of knee osteoarthritis, the extent of pain as reportedly experienced in the last week-a variable that shows an exponential increase over time and a risk factor for developing disability [30] was assessed, along with sleep, pain self-efficacy and depressive symptoms among other factors.

The finding that the degree of sleep experienced by the subject, which was compromised in more than 50 percent of respondents, was correlated with their pain was expected, but the further finding that self-efficacy scores for controlling pain when sleeping - that provided aggregated responses to the question 'How certain are you can keep arthritis pain from interfering with your sleep? also a significant correlate, is a novel observation.

Thus the hypotheses of this study were generally supported, despite obvious limitations, and may be important, because when examined more closely, this pain self-efficacy correlate relating to night pain was associated not only with sleep quality, but with depression, and pain.

Moreover, while these cross-sectional findings are clearly only exploratory, and cannot separate cause from effect, they are commensurate with findings of the negative impact of low self-efficacy [31], sleep disturbances [32], and pain [33] that increases with the severity of the disease [24].

In addition, while several other variables traditional measures were conducted, none were as closely linked to sleep as low pain self efficacy for offsetting night pain, a strong correlate of depression and pain. This latter finding that this only the item score on the arthritis pain self-efficacy scale was significantly correlated with pain, was observed in a larger study of both men and women [17]. It is also consistent with what we know about the increasing importance of both sleep and self-efficacy attributes, as well as depression in the context of ameliorating knee osteoarthritis. It also clearly draws attention in our view to the need to specifically examine the role of Lorig et al.'s arthritis pain self-efficacy survey items [16] independently, as well as collectively in both in research and practice contexts.

Other data from the sickle cell disease research [20] reveal that although some individuals (16.7\%) reported sleeping very well, the majority $(83.3 \%)$ do not sleep very well, and a greater number of individuals (93.3\%) reported having some pain, which is not dissimilar from those with painful arthritis, as outlined by Davis et al. [19]. Among those adults with chronic pain, Adegbola et al. [20] concluded that the concept of self-efficacy was of special importance in efforts to maintain a stable quality of health among the population.

Diaz-Pedro et al. [21] who studied fibromyalgia a painful muscle disease found subjective poor sleep quality among all their participants. In addition, pain correlated with subjective and objective sleep 
Sleep, Sleep Self-Efficacy Perceptions and Related Correlates in Women with Knee Osteoarthritis-An Exploratory Secondary Analysis

parameters, self-efficacy, anxiety, and, marginally, with depression. The mediated regression analysis suggested that the best models to explain the impact of pain on anxiety and depression included, as mediators, subjective sleep quality, objective sleep efficiency, and self-efficacy, which explained $34 \%$ of the variance, with objective sleep efficiency being the mediator with the highest influence.

More recently, a negative long term impact of poor self-efficacy on the mobility performance of women affected by knee osteoarthritis [22] has reinforced the importance of this construct in the context of osteoarthritis, while other research continues to indicatesleepisassociated withaltered pain processing, which varies by gender [23], and is more likely to be problematic in those osteoarthritis cases with higher numbers of comorbid conditions, cardiovascular diseases, pain in more than one joint, disease severity [24], and those in low versus high health status. The latter health issues are more than likely to prevail among the older adult with osteoarthritis than not, thus drawing further attention to the possible clinical salience of the current data. They also draw attention to the fact that for advancing the ability of the older adult to self-manage their osteoarthritis, while minimizing their disability, factors other than the mainstream physical correlates of the disease should be regularly assessed and targeted as implied by Nazarinasab et al. [25]. Indeed, it seems that if improvements in either sleep or the patient's ability to be more confident about controlling pain when trying to sleep-along with pain relief directives are employed as reasonable intervention points, especially in those with two or more pain sites [26], the potential to impact patients both physically speaking as well as cognitively speaking, especially in the presence of depression, poor self-rated health, and cardiovascular disease, the outcomes will undoubtedly be greatly increased as discussed by Viitiello et al. [27], Rosemann et al. [28], Wilcox et al . [35] Sugai et al. [36], Chen et al. [37] and Woolhead [38].

\section{CONCLUSION}

Knee joint osteoarthritis, a highly debilitating chronic health condition with no known cure, progressively affects the functional ability and life quality of many adults, especially women, extremely adversely. Although the risk factors for knee osteoarthritis have often been deemed to be largely neuromuscular or biomechanical in origin, rather than reflective of health behaviors and cognitive factors, the extent and quality of sleep, factors linked to sleep disturbances, such as obesity, pain self-efficacy and depression are increasingly being discussed as playing a possible mediating or moderating role in influencing the disease progression. In particular, recent research points to the fact that helping patients with knee osteoarthritis to have a good night's sleep may prove more helpful than not in efforts to reduce fatigue, associated depressive symptoms, as well as pain, and to maintain a healthy weight, but whether or not any of these factors differentially impact women or even present as significant correlates among women with typical disease characteristics is unknown.

This present exploratory analysis, while restricted to women and selected variables of interest, has clearly indicated that a regular assessment of sleep quality, and self-efficacy for controlling pain, especially at night, can potentially assist in efforts to better understand individual disease presentations, as well as to tailor recommendations more specifically. To establish if this linkage specifically affects women more than men, further research in this regard is strongly encouraged and may yet prove insightful in enlarging our understanding of knee osteoarthritis in women and men.

As knee osteoarthritis is a leading cause of increasing functional impairments in aging adults, and since it deteriorates slowly, rather than rapidly [29], and very few efficacious forms of intervention exist [34], it is possible that more standardized universal efforts to assess the knee osteoarthritis patient's pain levels, plus their sleep quality, and confidence for controlling night pain early on in the disease process, as well as carrying out periodic health checks, can potentially offset the rate and magnitude of any ensuing disability quite markedly. This is owing to the strong association observed in this study, even when other commonly recognized factors are considered, such as obesity, and the fact pain control self-efficacy at night is strongly associated with pain, depression, sleep difficulties, and overall pain self-efficacy.

However, until more research is forthcoming, it still seems that the importance of sleep and high selfefficacy for managing pain in the context of patient 
care should not be overlooked, especially among those with the most severe knee osteoarthritis who are subject to nocturnal pain increases and depression $[34,36,39,40]$.
The validity of the schematic representation of the correlates of sleep and pain self-efficacy shown below should also be carefully examined in other patient groups, including men.

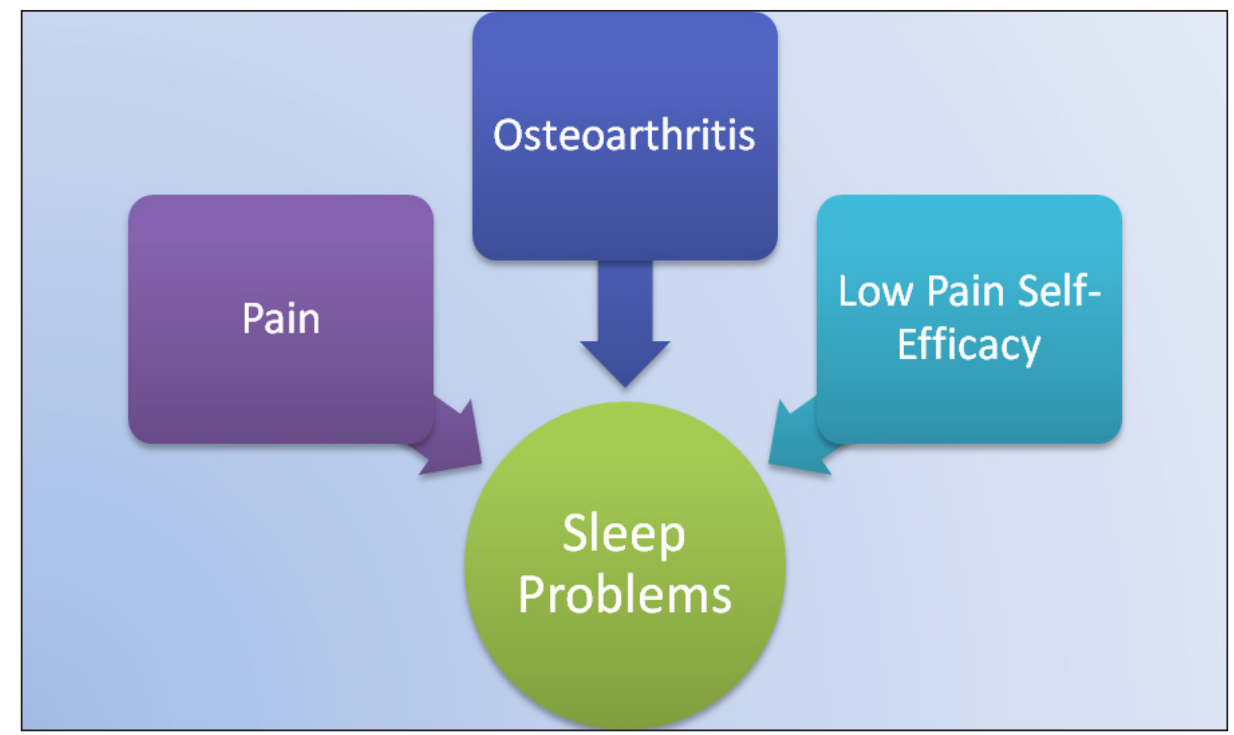

Figure 2. Schematic Representation of Interplay of Sleep and Knee Osteoarthritis

\section{REFERENCES}

[1] Vincent KR, Conrad BP, Fregly BJ, Vincent HK. The pathophysiology of osteoarthritis: a mechanical perspective on the knee joint. PM R. 2012;4(5 Suppl):S3-9. doi: 10.1016/j.pmrj.2012.01.020.

[2] Gresham GE, Rathey UK. Osteoarthritis in knees of aged persons. JAMA 1975;233:168-170.

[3] Omori G, Narumi K, Nishino K, Nawata A, Watanabe $\mathrm{H}$, Tanaka $\mathrm{M}$, Endoh $\mathrm{K}$, Koga $\mathrm{Y}$. Association of mechanical factors with medial knee osteoarthritis: a cross-sectional study from Matsudai Knee Osteoarthritis Survey. J Orthop Sci. 2016 Apr 14. pii: S0949-2658(16)30004-5. doi: 10.1016/j.jos.2016.03.006.

[4] Ozcakir S, Raif SL, Sivrioglu K, Kucukcakir N. Relationship between radiological severity and clinical and psychological factors in knee osteoarthritis. Clin Rheumatol. 2011;12:1521-1526.

[5] Theis KA, Helmick CG, Hootman, JM. Arthritis burden and impact are greater among U.S. women than men: intervention opportunities. J Womans Health. 2007;16:441-453.

[6] Petterson SC, Raisis L, Bodenstab A, SnyderMackler L. Disease-specific gender differences among total knee arthroplasty candidates. J Bone Joint Surg. 2007;89:2327-2333.

[7] Kawi J, Schuerman S, Alpert PT, Young D. Activation to self-management and exercise in overweight and obese older women with knee osteoarthritis. Clin Nurs Res. 2015;24(6):644660. Doi: $10.1177 / 1054773814544167$.

[8] Parmelee PA, Cox BS, DeCaro JA, Keefe FJ, Smith DM. Racial/ethnic differences in sleep quality among older adults with osteoarthritis. Sleep Health. 2017;3:163-169. doi: 10.1016/j. sleh.2017.03.010.

[9] Plotnikoff R, Karunamuni N, Lytvyak E, Penfold C, Schopflocher D, Imayama I, Johnson ST, Raine K. Osteoarthritis prevalence and modifiable factors: a population study. BMC Public Health. 2015;15:1195. doi: 10.1186/s12889-015-2529-0.

[10] Dekker J, Van Dijk GM, Veenhof C. Risk factors for functional decline in osteoarthritis of the hip or knee. Curr Opin Rheumatol. 2009;21:520-524.

[11] Watanabe H, Urabe K, Takahira N, Ikeda N, Fujita M, Obara S, Hendona T, Aikawa J, Itoman M. Quality of life, knee function, and physical activity in Japanese elderly women with early- 
Sleep, Sleep Self-Efficacy Perceptions and Related Correlates in Women with Knee Osteoarthritis-An Exploratory Secondary Analysis

stage knee osteoarthritis. J Orthop Surg (Hong Kong). 2010;18(1):31-34.

[12] Yeung WK, Morgan K, Mckenna F. Comparison of sleep structure and psychometric profiles in patients with fibromyalgia, osteoarthritis and healthy controls. J Sleep Res. 2018;27:290-298. doi: $10.1111 /$ jsr.12588.

[13] Meenan RF, Gertman PM, Mason JH, Dunaif R. The Arthritis Impact Measurement Scales: further investigation of a health status measure. Arthritis Rheum. 1982;25:1048-1053.

[14] Radloff LS. The CES-D scale: a self report depression scale for research in the general population. Appl Psychol Measurement. 1977;1:385-401.

[15] Scott J, Huskinsson EC: Graphic representation of pain. Pain 1976;2:175-184.

[16] Lorig K, Chastain RL, Ung E, Shoor S, Holman HR. Development and evaluation of a scale to measure self-efficacy in people with arthritis. Arthritis Rheum. 1989;32:37-44.

[17] Marks R. Physical and psychological correlates of disability among a cohort of individuals with knee osteoarthritis. Can J Aging. 2007;26:367377. doi: 10.3138/cja.26.4.367.

[18] Doğan N, Göriş S, Demir H. [Levels of pain and self-efficacy of individuals with osteoarthritis]. Agri. 2016; 28(1): 25-31. doi: 10.5505/ agri.2015.30085.

[19] Davis GC. Improved sleep may reduce arthritis pain. Holist Nurs Pract. 2003;17(3):128-135.

[20] Adegbola M. Sleep Quality, pain and selfefficacy among community-dwelling adults with sickle cell disease. J Natl Black Nurses Assoc. 2015;26(1):15-21.

[21] Diaz-Piedra C, Catena A, Miro E, Martinez MP, Sanchez AI, Buela-Casal G. The impact of pain on anxiety and depression is mediated by objective and subjective sleep characteristics in fibromyalgia patients. Clin J Pain. 2014;30(10):852-859. doi: 10.1097/AJP.0000000000000040

[22] Brisson NM, Gatti AA, Stratford PW, Maly MR. Selfefficacy, pain, and quadriceps capacity at baseline predict changes in mobility performance over
2 years in women with knee osteoarthritis. Clin Rheumatol. 2018;37(2):495-504. doi: 10.1007/ s10067-017-3903-3

[23] Petrov ME, Goodin BR, Cruz-Almeida Y, King C, Glover TL, Bulls HW, Herbert M, Sibille KT, Bartley EJ, Fessler BJ, Sotolongo A, Staud R, Redden D, Fillingim RB, Bradley LA. Disrupted sleep is associated with altered pain processing by sex and ethnicity in knee osteoarthritis. J Pain. 2015;16(5):478-90. doi: 10.1016/j. jpain.2015.02.004.

[24] Sasaki E, Tsuda E, Yamamoto Y, Maeda S, Inoue R, Chiba D, Okubo N, Takahashi I, Nakaji S, Ishibashi Y. Nocturnal knee pain increases with the severity of knee osteoarthritis, disturbing patient sleep quality. Arthritis Care Res. 2014;66:1027-1032. doi: 10.1002/acr.22258.

[25] Nazarinasab M, Motamedfar A, Moqadam AE. Investigating mental health in patients with osteoarthritis and its relationship with some clinical and demographic factors. Reumatologia. 2017;55:183-188. doi: 10.5114/ reum.2017.69778.

[26] Christensen JO, Johansen S, Knardahl S. Psychological predictors of change in the number of musculoskeletal pain sites among Norwegian employees: a prospective study. BMC Musculoskelet Disord. 2017;18(1):140. doi: 10.1186/s12891-017-1503-7.

[27] Vitiello MV, McCurry SM, Shortreed SM, Baker LD, Rybarczyk BD, Keefe FJ, Von Korff M. Shortterm improvement in insomnia symptoms predicts long-term improvements in sleep, pain, and fatigue in older adults with comorbid osteoarthritis and insomnia. Pain 2014;155:15471554. doi: 10.1016/j.pain.2014.04.032.

[28] Rosemann T, Laux G, Kuehlein T. Osteoarthritis and functional disability: results of a cross sectional study among primary care patients in Germany. BMC Musculoskelet Disord. 2007;8:79.

[29] van Dijk GM, Dekker J, Veenhof C, van den Ende $\mathrm{CH}$; Carpa Study Group. Course of functional status and pain in osteoarthritis of the hip or knee: a systematic review of the literature. Arthritis Rheum. 2006;55(5):779-785. 
Sleep, Sleep Self-Efficacy Perceptions and Related Correlates in Women with Knee Osteoarthritis-An Exploratory Secondary Analysis

[30] Gibson SJ, Lussier D. Prevalence and relevance of pain in older persons. Pain Med. 2012; Suppl 2:S23S26. doi: 10.1111/j.1526-4637.2012.01349.x

[31] Issa SN, Sharma L. Epidemiology of osteoarthritis: an update. Curr Rheumatol. Rep. 2006;8(1):7-15.

[32] Leigh TJ, Hindmarch I, Bird HA, Wright V. Comparison of sleep in osteoarthritic patients and age and sex matched healthy controls. Ann Rheum Dis. 1988;47(1):40-42.

[33] Jung JH, Seok H, Choi SJ, Bae J, Lee SH, Lee MH, Kim JH, Song GG. The association between osteoarthritis and sleep duration in Koreans: a nationwide cross-sectional observational study. Clin Rheumatol. 2018;37:1653-1659. doi: 10.1007/s10067-018-4040-3.

[34] Newberry SJ, FitzGerald J, SooHoo NF, Booth M, Marks J, Motala A, Apaydin E, Chen C, Raaen L, Shanman R, Shekelle PG. Treatment of osteoarthritis of the knee: an update review [internet]. Rockville (MD): Agency for Healthcare Research and Quality (US); 2017 May. Available from http://www.ncbi.nlm.nih.gov/books/ NBK447543/

[35] Wilcox S, Brenes GA, Levine D, Sevick MA, Shumaker SA, Craven T. Factors related to sleep disturbance in older adults experiencing knee

pain or knee pain with radiographic evidence of knee osteoarthritis. J Am Geriatr Soc. 2000;48(10):1241-1251.

[36] Sugai K, Takeda-Imai F, Michikawa T, Nakamura T, Takebayashi T, Nishiwaki Y. Association between knee pain, impaired function, and development of depressive symptoms. J Am Geriatr Soc. 2018;66(3):570-576. doi: 10.1111/jgs.15259.

[37] Chen CJ, McHugh G, Campbell M, Luker K. Subjective and objective sleep quality in individuals with osteoarthritis in Taiwan. Musculoskeletal Care. 2014 Dec 10. doi: 10.1002/msc.1094.

[38] Woolhead G, Gooberman-Hill R, Dieppe P, Hawker G. Night pain in hip and knee osteoarthritis: a focus group study. Arthritis Care Res. 2010;62(7):944949. doi: 10.1002/acr.20164.

[39] Parmelee PA, Tighe CA, Dautovich ND. Sleep disturbance in osteoarthritis: linkages with pain, disability, and depressive symptoms. Arthritis Care Res. 2015:67(3):358-365. doi: 10.1002/ acr.22459.

[40] Taylor-Gjevre RM, Giever JA, Nair B, Skomro R, Lim HJ. Components of sleep quality and sleep fragmentation in rheumatoid arthritis and osteoarthritis. Musculoskeletal Care. 2011;9(3): 152-159. doi: 10.1002/msc.208.

Citation: Dr. Ray Marks. Sleep, Sleep Self-Efficacy Perceptions and Related Correlates in Women with Knee Osteoarthritis-An Exploratory Secondary Analysis. Open Journal of Geriatrics. 2018; 1(1): 21-30.

Copyright: (C) 2018 Dr. Ray Marks. This is an open access article distributed under the Creative Commons Attribution License, which permits unrestricted use, distribution, and reproduction in any medium, provided the original work is properly cited. 